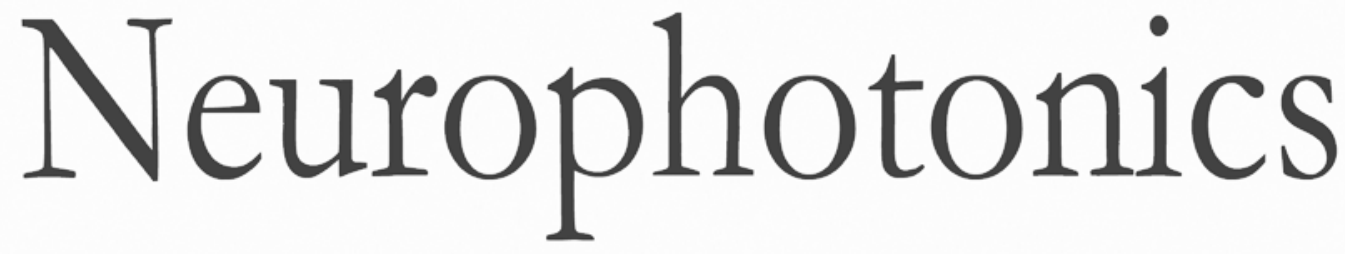

\title{
Review of the potential of optical technologies for cancer diagnosis in neurosurgery: a step toward intraoperative neurophotonics
}

Fartash Vasefi

Nicholas MacKinnon

Daniel L. Farkas

Babak Kateb 


\title{
Review of the potential of optical technologies for cancer diagnosis in neurosurgery: a step toward intraoperative neurophotonics
}

\author{
Fartash Vasefi, ${ }^{a, b, c, *}$ Nicholas MacKinnon, ${ }^{a}$ Daniel L. Farkas, ${ }^{\text {a,d }}$ and Babak Kateb ${ }^{b, c, e, f, *}$ \\ ${ }^{a}$ Spectral Molecular Imaging Inc., 13412 Ventura Boulevard, Suite 250, Sherman Oaks, California 91423, United States \\ 'Brain Mapping Foundation, 8159 Santa Monica Boulevard, Suite 200, West Hollywood, California 90046, United States \\ 'Society for Brain Mapping and Therapeutics (SBMT), 8159 Santa Monica Boulevard, Suite 200, West Hollywood, California 90046, United States \\ dUniversity of Southern California, Department of Biomedical Engineering, 1042 Downey Way, Los Angeles, California 90089, United States \\ ${ }^{e}$ California Neurosurgical Institute, 25751 McBean Pkwy \#305, Santa Clarita, California 91355, United States \\ 'National Center for Nano-Bio-Electronics (NCNBE), NASA Research Park, P.O.Box 23, Moffett Field, California 94035, United States
}

\begin{abstract}
Advances in image-guided therapy enable physicians to obtain real-time information on neurological disorders such as brain tumors to improve resection accuracy. Image guidance data include the location, size, shape, type, and extent of tumors. Recent technological advances in neurophotonic engineering have enabled the development of techniques for minimally invasive neurosurgery. Incorporation of these methods in intraoperative imaging decreases surgical procedure time and allows neurosurgeons to find remaining or hidden tumor or epileptic lesions. This facilitates more complete resection and improved topology information for postsurgical therapy (i.e., radiation). We review the clinical application of recent advances in neurophotonic technologies including Raman spectroscopy, thermal imaging, optical coherence tomography, and fluorescence spectroscopy, highlighting the importance of these technologies in live intraoperative tissue mapping during neurosurgery. While these technologies need further validation in larger clinical trials, they show remarkable promise in their ability to help surgeons to better visualize the areas of abnormality and enable safe and successful removal of malignancies. ( The Authors. Published by SPIE under a Creative Commons Attribution 3.0 Unported License. Distribution or reproduction of this work in whole or in part requires full attribution of the original publication, including its DOI. [DOI: 10.1117/1.NPh.4.1.011010]
\end{abstract}

Keywords: neurophotonics; optical imaging; brain tumor; image-guided therapy; intraoperative brain mapping.

Paper 16053VSSR received Jul. 23, 2016; accepted for publication Nov. 7, 2016; published online Dec. 26, 2016.

\section{Introduction}

Today, nearly 700,000 people in the United States are living with a primary brain tumor, and $\sim 78,000$ more will be diagnosed in 2016. ${ }^{1}$ Glioblastoma multiforme (GBM) is the most common infiltrating primary brain tumor in adults and one of the most aggressive cancers with sever negative effect on patient and health system. ${ }^{2}$ Many other brain tumors (such as meningiomas) are noninfiltrating and can be removed without damaging normal brain tissue. GBMs represent $50 \%$ of gliomas and $16 \%$ of all primary brain tumors, ${ }^{3-5}$ but despite intense multipronged therapy consisting of surgery, radiation, and chemotherapy, the outcome of patients with GBM remains poor, ${ }^{6}$ with 5-year survival rates of $<10 \%$. ${ }^{4,5}$ Resistance to these therapies is most likely caused by several factors, including the blood-brain barrier (BBB) that blocks therapeutic agents from the tumor site ${ }^{3,7-12}$ and the chemo-resistant and radiation-resistant nature of glioma-initiating cells. GBMs also create a hostile immunosuppressive tumor microenvironment that prevents the inducement of anti-GBMspecific immune responses. ${ }^{7-12}$ Overall, tumor delineation is an extremely difficult and subjective process, requiring a skilled neurosurgeon to interpret sometimes inaccurate preoperative imaging to achieve near-complete resection. Because of postimaging changes that occur during procedures, there is an ongoing need for real time, intraoperative detection.

\footnotetext{
${ }^{*}$ Address all correspondence to: Fartash Vasefi, E-mail: fvasefi@opmol.com fartash.vasefi@WorldBrainMapping.Org; Babak Kateb, E-mail: Babak. Kateb@WorldBrainMapping.Org
}

When considering the resection of a tumor, one must be aware of the plastic nature of the human brain. Brain tissue is extremely flexible and deforms following dural opening. ${ }^{13}$ This phenomenon, known as "brain shift," is affected by many factors such as mechanical tumor resection, evacuation of cystic components, gravity, pharmacologic responses, osmotic shifts, drainage of cerebrospinal fluid, and other surgical effects. ${ }^{14-17}$ Brain shift leads to significant challenges in the use of conventional neuronavigational systems, which use preoperatively acquired images and stereotaxy to depict the anatomical location and estimate the three-dimensional (3-D) extent of brain tumors. ${ }^{13,18}$ Currently, there are two main nonoptical technologies that have been implemented to overcome the problem of brain shift during neurosurgery: intraoperative magnetic resonance imaging (MRI) (ioMRI) ${ }^{19,20}$ and intraoperative Ultrasound (ioUS). However, many optical technologies are also beginning to be applied in this context, including infrared (IR) thermal imaging, Raman spectroscopy, fluorescence spectroscopy and imaging, fluorescence lifetime spectroscopy and imaging, and optical coherence tomography (OCT). Here, we present a summary of clinical implementations of these optical technologies, as intraoperative guidance tools in human neurosurgery, with emphasis on their respective merits and challenges.

\section{Nonoptical Technologies}

\subsection{Intraoperative $\mathrm{MRI}$}

ioMRI-guided intracranial surgery was first applied in late 1990s. ${ }^{21,22}$ High-field MRI with an integrated neuronavigation 
system is one of the most advanced nonoptical intraoperative imaging methods. ${ }^{23}$ It allows for objective control of the extent of resection and for immediate update of the neuronavigation data. ${ }^{24,25}$ Anatomical and functional information enable the surgeon to decide whether it is necessary and safe to proceed with tumor resection. IoMRI allows more precise delineation of the tumor margin and detects hemorrhage. ${ }^{26,27}$ It also provides an objective measure of the needed extent of surgery and allows more radical, yet safe, tumor removal, regardless of tumor type. ${ }^{28}$ Its advantages over ultrasound or CT-scan-guided resections include lack of ionizing radiation, superior multiplanar imaging, and user independence. ${ }^{29}$ IoMRI is rapidly becoming the procedure of choice for many neurosurgeons because of advantages of real-time high-quality images and ability to account for brain shift. However, this technique contains many disadvantages as well, including cost and time constraints. IoMRI systems are usually too expensive for most neurosurgical departments, involve a time-consuming image acquisition process, and require expensive nonmagnetic surgical tools. ${ }^{13}$ Furthermore, using these techniques, it is not possible to track surgical instruments as they pass through brain tissue to cysts or tumors. ${ }^{30}$ Finally, after resection of the tumor and the resulting brain shift, the interpretation of the MRI data can still be difficult. The use of ioMRI for neurosurgical procedures also involves a gradual learning curve to acquire sufficient expertise, ${ }^{28}$ and if intraoperative imaging suggests there is residual tumor present, then surgeons must apply their own clinical experience and expertise to each specific situation.

\subsection{Intraoperative Ultrasound}

ioUS technology is useful because it is a real-time imaging system that remains mostly unaffected by brain shift. It is also very easy to handle and inexpensive, two important advantages over ioMRI. ${ }^{31-33}$ The two-dimensional (2-D) US image acquisition is significantly faster than ioMRI and scans can be performed multiple times during surgery, without major interruption of the workflow. ${ }^{24}$ US imaging can be also integrated with neuronavigation systems. ${ }^{34}$ Today the technology and image quality of ultrasound have been improved significantly, providing the surgeon with a useful way to identify tumors and make a preliminary classification of low-grade or high-grade glioma. ${ }^{35}$ Its sensitivity and specificity in detecting larger or deeply located tumor remnants are comparable to those of high-field ioMRI. ${ }^{36}$ Some studies have even reported the ability of ultrasound to differentiate edema from solid tumor, a task that is difficult for both CT and MR imaging. ${ }^{37}$ However, two shortcomings are that ioUS provides lower sensitivity and specificity in the detection of small or superficial tumor remnants. ${ }^{24}$ Furthermore, the image acquisition and interpretation are subjective and require adequate training and experience. ${ }^{24}$ Other confounding factors that may affect the interpretation of the ioUS image are the presence of dropouts from blood, cottonoids, or proteinaceous fluid, which can all reduce the ultrasound image quality, or the contrast of the disrupted $\mathrm{BBB} .{ }^{31}$

\section{Optical Measurements of Brain Tissue}

When light impinges upon a tissue, it can be transmitted, absorbed, reflected/scattered, and re-emitted as fluorescence, or have its energy altered by Raman scattering. Spectroscopy measures the wavelength dependency of these phenomena to determine important properties of the tissue being probed. Depending on the energy of the light and the nature of the target tissue, some of these interactions, such as absorption by particular chemical bonds, can reflect the presence and quantity of well-understood tissue constituents and aid in tissue discrimination. ${ }^{38,39}$

Optical imaging and spectroscopy have evolved from ancillary research tools to various diagnostic applications. Optical imaging, by far the oldest of bioimaging methods, helps us understand better by seeing better. Technologies have been developed to build on the intrinsic capabilities of the human eye and human cognition. The first multilens microscopes for medical research were built about 400 years ago and have been used to extend the spatial resolution limits of human vision. ${ }^{40}$ Optical filters and wavelength selection technologies have extended the wavelength resolution of human vision. ${ }^{41}$ With the advent of digital imaging, scientists have been able to harness the full power of these technologies by using computational tools for image processing and analysis. Modern optical imaging is special in several ways: (a) it is intuitive and noninvasive; (b) it covers many orders of magnitude; in time from femtoseconds to seconds, in energetic intensity from tens of watts/joules to a few pico-watts/joules, and in space from meters down to fractions of a micrometer, while having very high resolution in each of these domains. Most importantly, given the diversity of biology, optical imaging is ideally suited to look, quantitatively, at the basic unit of life (the cell), while alive, in realistic environments (either in vivo or in laboratory conditions approximating this). ${ }^{42}$ When light interacts with tissue, its bulk properties can be modified. These include changes in: (1) optical intensity due to absorption, (2) polarization, timing, and coherence due to multiple scattering, (3) wavelength distribution due to chromophore absorption or fluorescence, and (4) interference due to parallel microstructures and optical index mismatches. These changes can be specific to different tissue types and can provide measurable image contrast that provides information that can be complementary and even synergetic. ${ }^{43}$

\subsection{Thermal Imaging}

Intraoperative thermal imaging (ioTI) is a neuroimaging technique that has the potential for locating the margins of brain tumors. The real-time anatomical and pathophysiological information provided by ioTI may contribute to safely improving the extent of tumor resection. ${ }^{44}$ The presence of a tumor often alters the homeostasis of the surrounding tissue and ioTI measurements are sensitive to this change. Changes in homeostasis that affect temperature can be due to low density in tumor microvessels, ${ }^{45,46}$ edema in the surrounding tissues due to increases in the volume of white matter caused by tumor growth, ${ }^{45,46}$ lower metabolism in the cortex overlying intracranial tumors, due to the "disconnection effect" caused by edema, ${ }^{45,46}$ or tumor necrosis, typically caused by reduction of tumor vasculature and metabolic activity.

Although not as common as ioMRI, ioTI has been used in several experimental models and case reports for brain tumor detection. In both animal and human models, glial-derived brain tumors display a consistently lower temperature than the surrounding normal tissue. ${ }^{45,46}$ In patients, Gorbach et al. ${ }^{46}$ showed that these tumors generally have a decrease of 0.5 to $2.0^{\circ} \mathrm{C}$ compared to normal surrounding tissue. Kateb et al. showed a case study of a 76-year-old female patient with metastatic intracortical melanoma. The data revealed a clear demarcation of tumor with significant temperature differences, up to $3.3^{\circ} \mathrm{C}$, between the tumor core $\left(36.4^{\circ} \mathrm{C}\right)$ and the surrounding 
normal tissue $\left(33.1^{\circ} \mathrm{C}\right)$ when intraoperatively measured by a ThermaCAM P60 FLIR system (wavelength sensitivity: 7.5 to $13 \mu \mathrm{m}){ }^{44}$

Thermal imaging provides a surface recording of a tissue's heat profile with low penetration, so the use of ioTI requires clear exposure of the lesion. Neural activity, local metabolism, blood flow, and thermal conductivity are all factors that contribute to the thermal profile of a tissue. ioTI is a versatile and useful intraoperative tool that is cost-efficient and noninvasive. Limitations of this technology include a lack of software that can autodelineate the tumor margin, a need for cameras with higher spatial resolution, and limited tissue penetration depth $(<0.5 \mathrm{~mm}){ }^{44}$ Ultimately, infrared thermography could become a vital addition to high-resolution neurosurgical microscopes that can accurately distinguish between normal and cancerous tissue using diverse multimode technologies.

\subsection{Raman Spectroscopy}

Raman spectroscopy is a nondestructive spectroscopic technique, based on the vibrational properties of the constituent molecules, that provides molecular information about the tissue under examination. ${ }^{47}$ The Raman signal results from tissue molecules being excited by a small amount of incident light at a specific wavelength. The remitted light has some of its photons shifted to different wavelengths as by the addition or subtraction of vibrational energy from some of the tissue intramolecular bonds. ${ }^{48}$ Contrast is achieved when the tissue molecular constituents differ enough that the Raman signals from two tissues have different wavelength distributions. Raman spectroscopy may be an ideal tool to intraoperatively delineate gray matter from white matter because it is minimally influenced by water content and can be performed in near-real time with sterile optical probes that a surgeon can apply to tissue in the operative field. $^{47}$

Raman spectroscopy imaging techniques have been used to identify histologic features in brain tissue frozen sections of rat glioma. The rat glioma model has similar morphology to glioblastoma multiforme. ${ }^{49}$ This study showed that Raman spectral features could be used to differentiate tumor, edema, necrotic, and healthy brain tissue. Specific spectral bands associated with proteins and lipids are the main Raman spectral features used for differentiation.

Recently, an intraoperative Raman-based spectroscopy technique detected invasive brain cancer in $<1 \mathrm{~s}$ during surgery and detected grade 2 to grade 4 gliomas in vivo ${ }^{50,51}$ It distinguished brain tissue invaded by cancer from normal brain tissue with a sensitivity of $93 \%$ and specificity of $91 \%$ (17 patients). The point spectroscopy method used a handheld fiber probe illuminating a 0.5 -mm-diameter tissue area. The probe sampled depth up to $\sim 1 \mathrm{~mm}$ with acquisition time for each measurement requiring about $0.2 \mathrm{~s}$. These Raman signatures are based on lipid bands (700 to $1142 \mathrm{~cm}^{-1}$ ), nucleic acid bands (1540 to $1645 \mathrm{~cm}^{-1}$ ), and the phenylalanine band in proteins

(a)

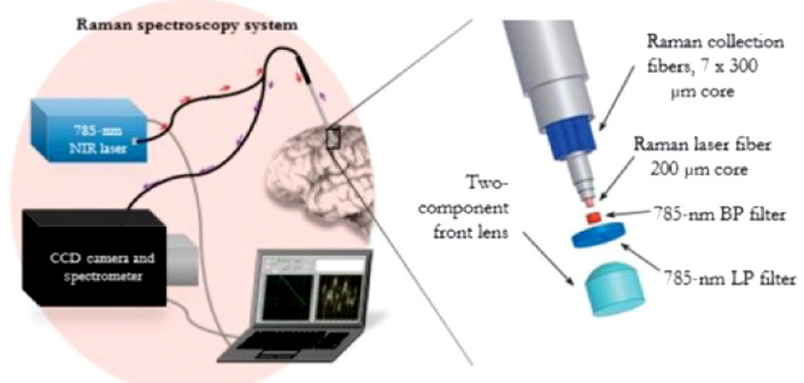

(b)

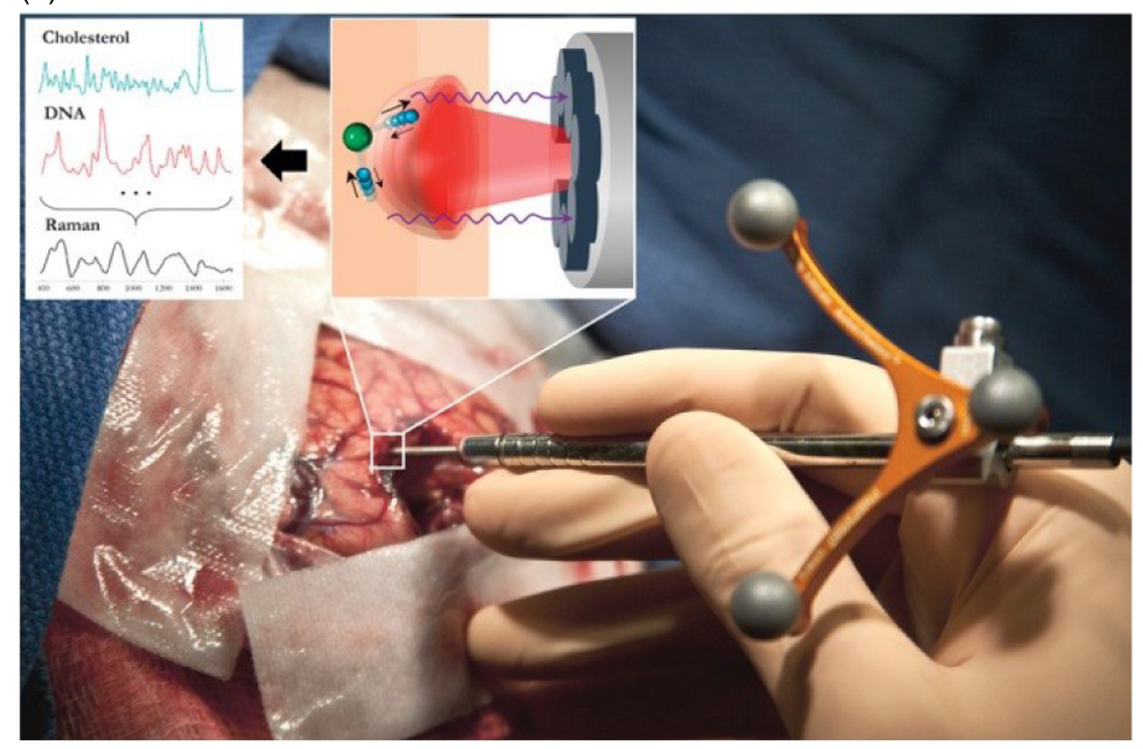

Fig. 1 Handheld contact fiber optic probe for Raman spectroscopy. (a) Experimental setup diagram. (b) A simple molecular vibrational mode is conceptually depicted (individual atoms in blue and green) interacting with the laser light (in red) to produce Raman scattering (in purple). 
$\left(1005 \mathrm{~cm}^{-1}\right)$. Machine learning algorithms created a classifier to differentiate between normal and tumor tissue types.

As shown in Fig. 1(a), the probe contained optical fibers connected to a NIR spectrum-stabilized laser $(\lambda=785 \mathrm{~nm})$ for inject energy and a fast CCD spectrometer for detection of the returning Raman signal. During each resection, the Raman signal was measured at several points in the surgical cavity [Fig. 1(b)].

Figure 2(a) shows a 3-D preoperative MRI of a patient with a grade 2 glioma, with dots identifying the Raman measurement locations (blue for normal and yellow for tumor). For three of the locations, the 2-D MRI slices are provided with corresponding pathology images, as well as the Raman spectra [Fig. 2(b)] for dense cancer, invasive cancer, and normal brain. Sample Raman spectra, preoperative MRI, and pathology images are also provided for a patient with a grade 3 glioma [Figs. 2(a) and 2(b)] and a patient with a grade 4 glioma [Fig. 2(C)].

Cancer cells showed some differences in various bands including (1) the lipid bands at 700 and $1142 \mathrm{~cm}^{-1}$ corresponding to cholesterol and phospholipids (26), (2) an increase from 1540 to $1645 \mathrm{~cm}^{-1}$, corresponding to a higher nucleic acid content, and (3) an increase at the $1005 \mathrm{~cm}^{-1}$ band, corresponding to phenylalanine breathing mode (26).

The new field of Raman-based optical biopsy for surgical guidance provides minimal disruption to workflow. Current development of more sensitive detectors and fast electronics shows the promise to overcome the low signal-to-noise ratio of the Raman signal, leading to Raman-based imaging. Future work will continue to explore Raman signal uses for brain tissue discrimination and surgical guidance in larger clinical trials.

\subsection{Optical Coherence Tomography}

OCT is a noninvasive, label-free, and cost-effective technique capable of imaging tissues in 3-D in near real time. ${ }^{52}$ OCT has spatial resolution in the micrometer range for depths up to a few millimeters. Clinical applications of OCT have been mainly in ophthalmology, however, over the last 20 years it has been extended to cancer detection in a variety of tissues. ${ }^{53,54}$ One of the first studies on ex vivo brain tissue by Bizheva et al. ${ }^{55}$ was reported in which the OCT signal was used to distinguish between healthy brain and multiple types of brain tumors. This study claims the source of OCT signal contrast comes from differences in cell nuclei, cysts, microvasculature, and microcalcifications in brain tissue.

Recently in 2015, structural and functional information provided by OCT has been explored to study brain diseases and tumors in vivo. ${ }^{56}$ In a study by Kut et al., OCT was used on ex vivo human tissue samples of 32 patients with grade 2 to 4 gliomas. They used attenuation-based thresholding to distinguish between normal and tumor tissue types. The threshold values were obtained by a researcher blinded to tissue type and grade. Specificities and sensitivities of $100 \%$ and $92 \%$ (for high-grade glioma) and $80 \%$ and $100 \%$ (for low-grade glioma) were reported.

In the same article, they have showed the extension of this work to an in vivo study with a cohort of nine subjects. They observed distortions in tissue microstructure and light attenuation profiles in the tumor region that were confirmed by biopsy and histopathological examination. The OCT system can measure an 8 - to $16-\mathrm{mm}^{3}$ tissue block in 1.2 to $2.4 \mathrm{~s}$, making it a useful tool with minimal disruption during neurosurgery.
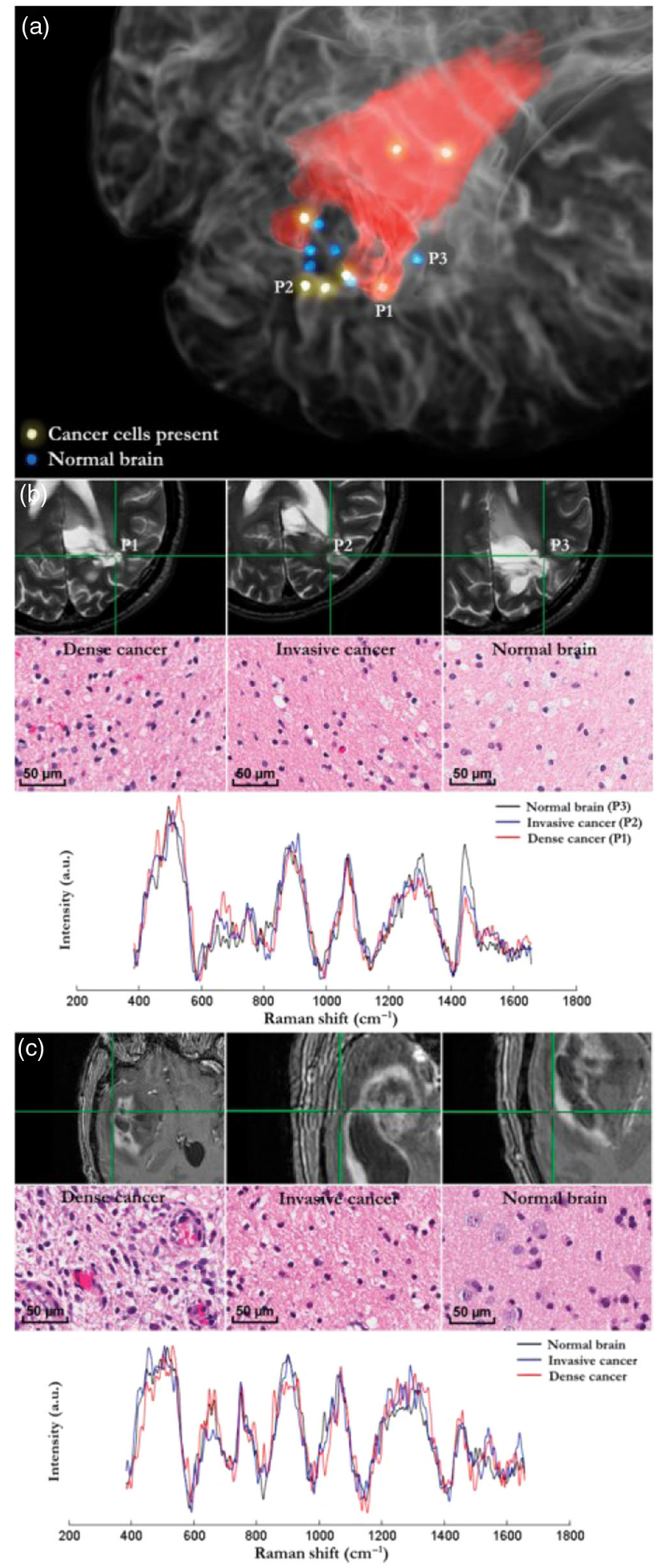

Fig. 2 Raman spectroscopy measurements colocated on preoperative MRl-grade 2 and 4 gliomas. (a) 3-D rendering of a preoperative T2-weighted MRI overlaid with the segmentation of a grade 2 astrocytoma in red. Samples for each tissue type are indicated by P1, P2, and P3. (b) Pathology images for regions P1 to P3 in (a). P1, P2, and $P 3$ are dense cancer, invasive cancer, and normal brain, respectively. The acquired Raman spectra are shown below. (c) In a different patient with grade $4 \mathrm{GBM}$, locations for dense cancer, invasive cancer, and normal brain are shown on a T1-weighted MRI with corresponding histopathology. 
(a)

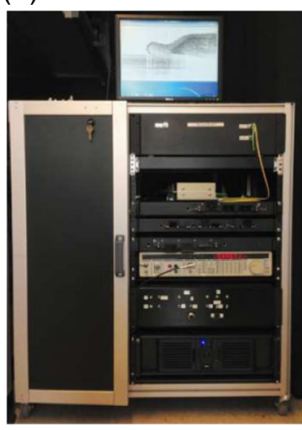

(b)

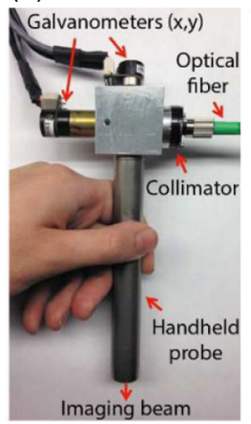

(c)

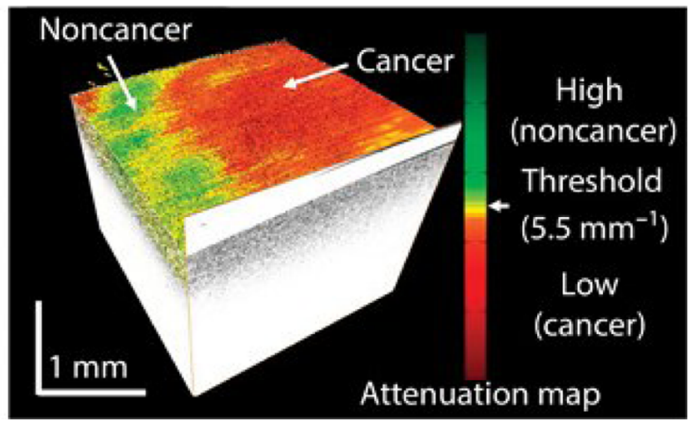

Fig. 3 (a) A SS-OCT system for intraoperative neurosurgery procedure. (b) A 2-D scanning handheld imaging probe. (c) Characteristic curves were determined using brain tissues in the training data set. A 3-D volumetric reconstruction map from the OCT images with an overlaid color-coded attenuation map distinguishing cancer and noncancer regions.

Figure 3(a) shows the swept-source OCT (SS-OCT) system and the handheld imaging probe that were used in the study. The OCT probe design offers a working distance of $25 \mathrm{~mm}$ and a confocal parameter of $0.3 \mathrm{~mm}$ [see Fig. 3(b)]. To remove the influence of depth-dependent effects on the beam profiles, they normalized the OCT signal from the tissue with the OCT signal from a phantom with known attenuation properties. The averaged OCT signal values of the biological tissue and phantom at different depths are used to calculate optical attenuation that provided the threshold parameter to differentiate between normal and cancerous tissues. An optimal attenuation threshold was established for identification of high-grade and low-grade human brain cancer. Finally, to facilitate potential intraoperative use of this technology, OCT images were displayed in 3-D and color-coded optical property maps showing tissue attenuation properties [Fig. 3(c)].

\subsection{Fluorescence-Based Technologies}

\subsubsection{Endogenous fluorescence in tissue}

There are several endogenous fluorophores that exhibit potential for analyzing tumor environments. These include amino acids such as tyrosine and tryptophan, structural proteins such as elastin and collagen, and enzyme cofactors [e.g., reduced nicotinamide adenine dinucleotide (NADH) and flavin-adenine dinucleotide (FAD)]. The altered metabolic state seen in brain tumors causes changes in these molecules' fluorescence signatures, which can be utilized for tissue characterization. By example, NADH concentration increases in tissues with higher metabolic activity. NADH displays fluorescence emission in the spectral range of 400 to $700 \mathrm{~nm}$ when exposed to UV radiation, whereas its oxidized form (NAD+) is nonfluorescent. In addition, the lifetime of NADH is either short or long, depending on the state of its protein binding sites. Changes in the ratio of free-to-bound NADH in tumor tissue results in fluctuations of the average lifetime of $\mathrm{NADH}^{57}$ Thus, both the intensity and lifetime of NADH fluorescence are considered to be potential indicators for monitoring the tumor microenvironment. Most endogenous fluorophores are excited by UV-VIS light (280 to $450 \mathrm{~nm}$ ) and emit fluorescence in the range of 370 to $700 \mathrm{~nm}$, as shown in Fig. 4. ${ }^{58,59}$ In general, tumor necrotic regions have lower fluorescence emission compared to normal tissue, under UV excitation at $355 \mathrm{~nm}$, likely due to lower tissue metabolism. One experimental approach demonstrated that the ratio of NADH:FAD fluorescence emission may play a significant factor in identifying brain tumors. ${ }^{60}$

\subsubsection{Steady-state fluorescence spectroscopy}

An autofluorescence study in a rat glioma model was presented by Chung et al. ${ }^{61}$ and it showed that there are relationships between brain tissue autofluorescence intensity and metabolic activity. For instance, fluorescence signals were lower in gliomas than in normal brain tissue, and flavin and porphyrin fluorescence in neoplastic tissues was different from that of normal tissue. Bottiroli et al. ${ }^{6}$ first reported an in vivo study on a limited number of patients that showed that brain tissue can be differentiated by its autofluorescence properties, including the emission peak and shape of fluorescence spectra, when excited at $366 \mathrm{~nm}$. Lin et al. reported a discrimination algorithm based on the ratio of the fluorescence emission and reflectance intensity at $625 \mathrm{~nm} .{ }^{58}$ Croce et al. ${ }^{63}$ reported more comprehensive ex vivo $(N=20)$ and in vivo $(N=12)$ spectrofluorometric analysis of brain tissue. They reported a fluorescence intensity

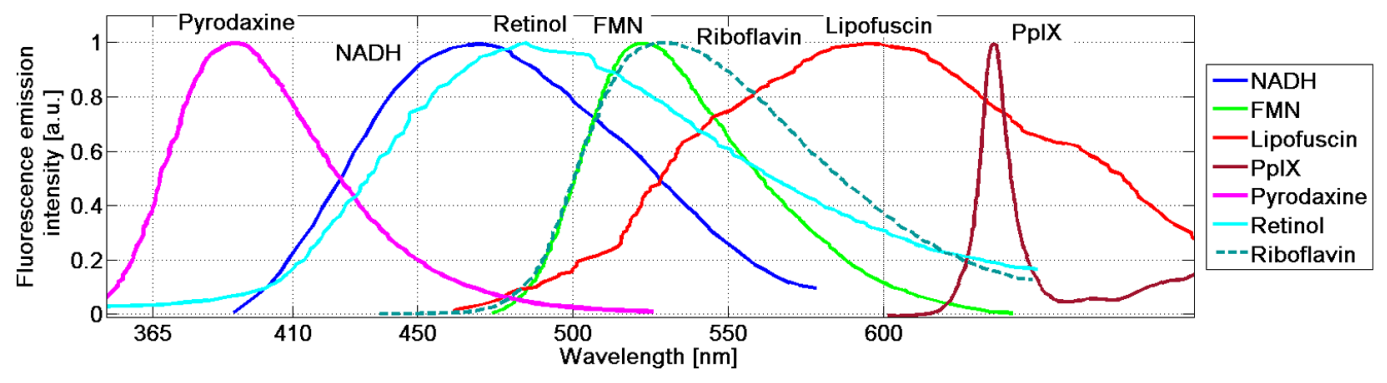

Fig. 4 Natural fluorophores of biological tissue. 
decrease and a broadening of the fluorescence emission band along with a redshift of the peak position in tumor compared to normal tissue, although some variability among the patients was observed.

The main advantage of steady-state fluorescence spectroscopy compared to time-domain fluorescence measurement is its simple, low-cost instrumentation that provides higher signal-to-noise ratios. The steady-state systems typically include one or more narrow band light sources and a grating-based spectrometer for collecting fluorescence emission. However, fluorescence spectroscopy is based on intensity measurements that can be affected by other factors such as tissue motion, high blood absorption, and photo-bleaching. These can add extra variability to the signal during surgery and decrease the classification specificity for tissue abnormalities. ${ }^{64}$ One of the approaches to overcome the limitations of steady-state fluorescence measurement is employing time-resolved fluorescence emission measurements (lifetime) as a main tissue differentiation factor. ${ }^{65}$

\subsubsection{Time-domain fluorescence measurement methods}

Time-resolved fluorescence determines the dynamics and intensity of fluorescence decay (lifetime). Fluorescence lifetime is the average time when a fluorophore remains in the excited state after being excited by the light source. It could be a more effective tool for optical biopsy because not only does the contrast from different fluorescence lifetimes result from changes of cellular or tissue environment, but also these changes are independent of fluorescent signal intensity, irregular brain surfaces, tissue illumination, blood, and fluorophore quantum yield. ${ }^{61,66}$ Time-resolved fluorescence measurement can also differentiate the fluorescence components of overlapping emission spectra, providing the ability to detect variations in $\mathrm{pH}$ and temperature. ${ }^{59,67}$ However, time-resolved fluorescence measurements (either spectroscopic or imaging) require more complex equipment, including more sophisticated lasers, high-speed detectors, and electronics that function in the picosecond range. A short-pulsed laser is widely used as the excitation light source for time-domain measurement, whereas a modulated light source is used for frequency-domain measurement. Singlepoint spectroscopy ${ }^{68-70}$ and multispectral/hyperspectral imaging systems ${ }^{71-73}$ have both been investigated for their potential use in tissue diagnosis. In the single-point method, a fiber optic probe delivers laser light and collects fluorescence signal, making time-resolved fluorescence spectroscopy (TRFS) much more compatible with the clinical setting. In imaging techniques, a single fiber or fiber bundles are used to collect the image in wide-field-based fluorescence-lifetime imaging microscopy (FLIM) ${ }^{74,75}$ whereas high-speed scanning modules (e.g., galvanometer mirrors) are used in scanning-based FLIM. ${ }^{72}$

Time-resolved fluorescence imaging. In this method, the fluorophore is excited by a short-pulse laser, whereas emission is detected at different times along the decay curve by a fixedgating time imaging detector (e.g., $200 \mathrm{ps}$ ), which is then used to reconstruct the decay curve in the time domain. With the use of a time-gated intensified charge-coupled device (ICCD), this method is suitable for obtaining an image over a large spatial area. Sun et al. reported the application of fluorescence lifetime imaging to glioblastoma detection during surgery. They reported weaker fluorescence emission intensity and longer lifetime at $460 \mathrm{~nm}$ for GBM compared to normal cortex for a limited number of patients $(N=3) .^{75}$ They evaluated the FLIM measurements with MRI imaging as well as pathology assessment. However, both the temporal resolution and the sensitivity were relatively low.

Time-resolved fluorescence spectroscopy. Time-resolved fluorescence spectroscopy (TR-FS) is a technique that uses ultrashort laser pulses to excite a tissue sample and record the corresponding fluorescence intensity decay in multiple spectral bands. ${ }^{76}$ The fluorescence decay is a robust measure of tissue characteristics and is not affected by the fluorescence intensity fluctuations commonly found in intraoperative data. This gives TR-FS an advantage over other optical techniques that can be susceptible to blood absorption, measurement distance from probes, and surgical microscope background light.

Butte et al. ${ }^{77}$ reported using TR-FS to detect fluorescence lifetime differences between normal brain tissue and meningioma in 22 patients with a sensitivity/specificity ratio of 89 / 100. Their statistical analysis was based on one-way analysis of variance (ANOVA) applied to spectroscopic parameters, such as the ratio between the various fluorescence intensities, and time-dependent parameters observed near the peak at $390 \mathrm{~nm}$. In 2011, they reported on the potential of applying TR-LIFS for intraoperative diagnosis of gliomas. ${ }^{78}$ Normal cortex $(N=35)$ and white matter measurements $(N=12)$ showed longer-lasting fluorescence emission at $390 \mathrm{~nm}$ compared with $460 \mathrm{~nm}$, whereas the 390-nm emission peak was absent in lowgrade gliomas and reduced in high-grade gliomas. The system required a relatively long data acquisition time $(\sim 30 \mathrm{~s})$ to capture each tissue measurement, which would need to be shortened substantially for effective integration in clinical workflows. ${ }^{79}$

Vasefi et al. ${ }^{80}$ recently reported on an improved TR-FS system with near real-time data acquisition and classification. In this study, tissue differentiation on larger tissue samples measured in vivo $\mathrm{NC}(n=10), \mathrm{WM}(n=12)$, and GBM $(n=13)$ with nine patients is described. The TR-FS system (shown in Fig. 5) used a 355-nm laser pulse (pulse width: $350 \mathrm{ps,} \mathrm{pulse}$ rate: $1 \mathrm{kHz}$ ) to excite tissue fluorescence and an optical demultiplexer to separate the emission photons into six wavelength bands from 360 to $700 \mathrm{~nm}$. Since multiple detectors would be costly and require individual calibration, each individual color band is passed through a fiber delay unit. The fiber delay units have fiber optic coils of varying lengths. The longer the fiber, the more delay occurs before the signal reaches the single system detector. This produces a train of signals containing

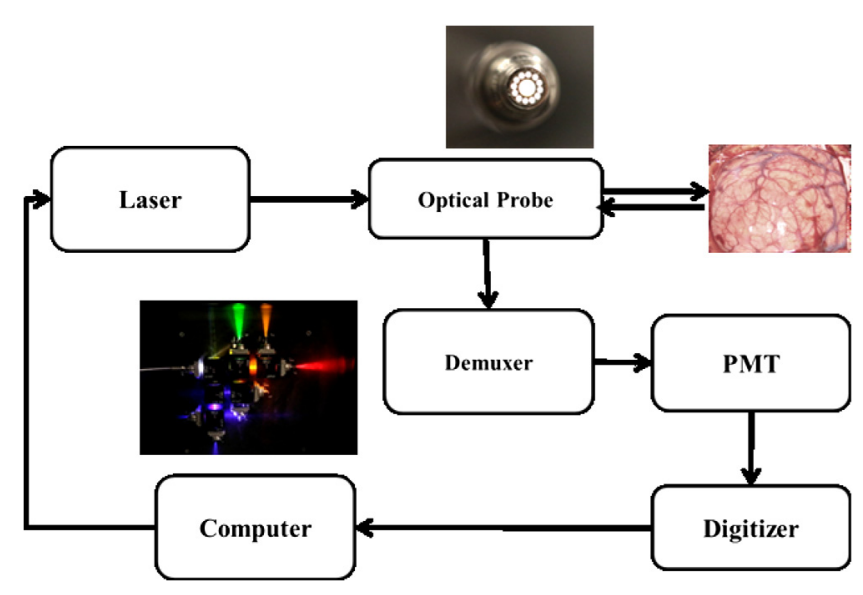

Fig. 5 TRFS system diagram. 
different color band information reaching the detector at different times.

The fluorescence decay function was calculated by deconvolving the fluorescence measurement by instrument response function. For each TR-FS measurement, six decay curves were measured corresponding to each color band. Since the fluorescence decay may contain multiple intrinsic fluorophores in tissue, the decay function cannot be presented by only average lifetime scale. An intrinsic fluorophore may have a monoexponential, biexponential, or multiexponential decay function.
Furthermore, there may be $>1$ intrinsic fluorophore that can further complicate the decay function analysis.

This method transforms the decay functions at different wavelengths into a 2-D spectro-lifetime matrix (SLM) with $m \times n$ dimensions, where $m$ is the number of spectral bands used in the measurements and $n$ is the number of decay points used. Figure 6 shows the three lifetime functions in six color bands corresponding to slow $\left(\tau_{[0.2]}\right)$, middle $\left(\tau_{[0.4]}\right)$, and fast $\left(\tau_{[0.6]}\right)$ decay components of fluorescence decay measured in human brain tissue. The lifetime values are separated by crossing

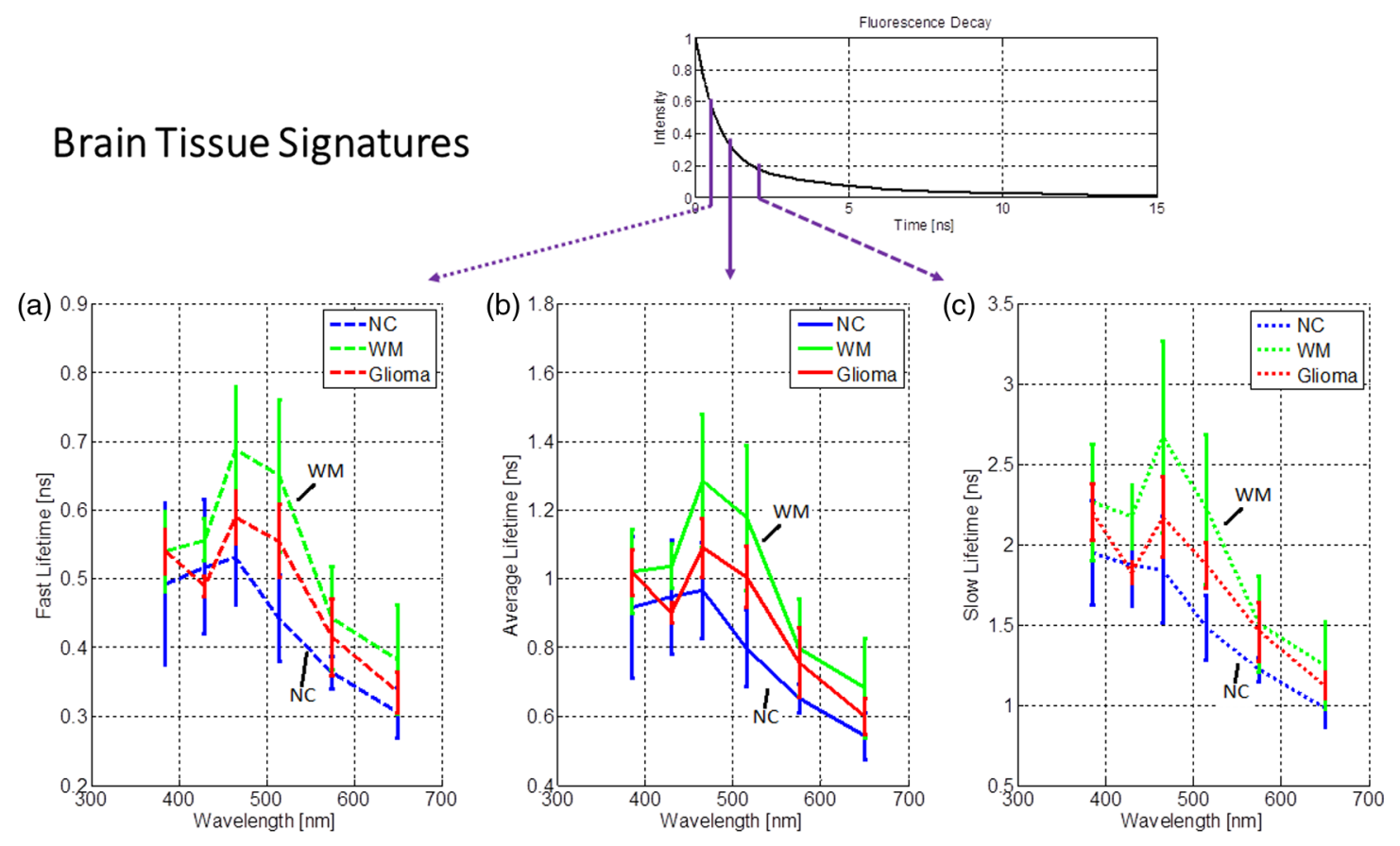

Fig. 6 (a) Spectral signatures of fast, (b) normal, and (c) slow lifetime for brain tissue measured by TRFS system.
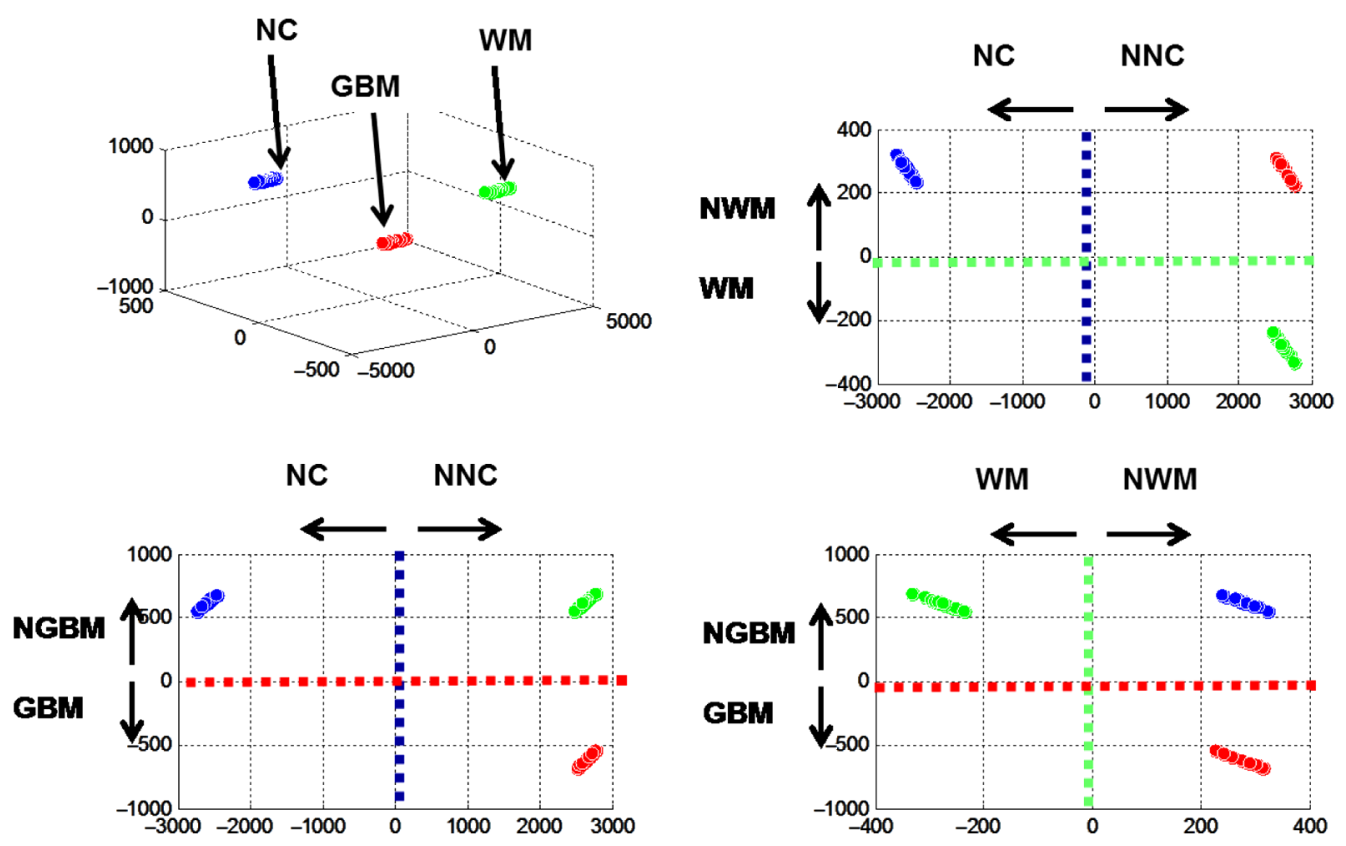

Fig. 7 TRFS classification output for differentiating normal cortex, white matter, and GBM tissue types. 


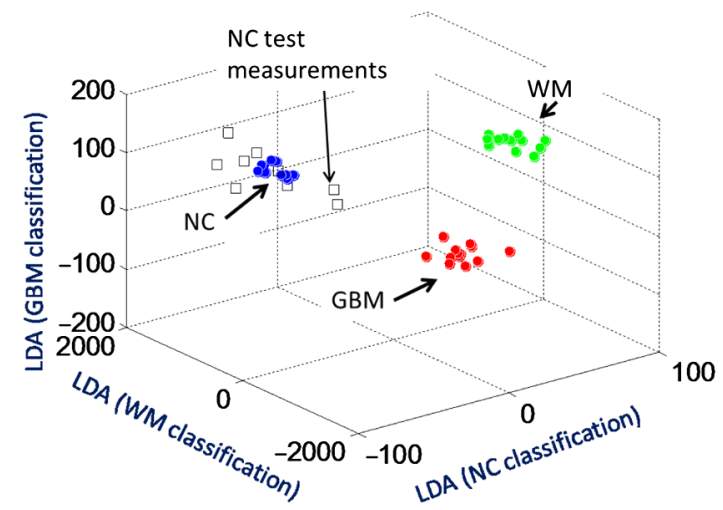

Fig. 8 Meningioma case study with normal cortex measurements $(N=8)$.

normalized fluorescence impulse response function at $0.2,0.4$, and 0.6 intensity levels.

Figure 7 shows classification results distinguishing NC, WM, and GBM measurements acquired in vivo. Linear discriminant analysis with a three-group subclassifier was implemented. The first subclassifier identified whether the TR-FS measurement is NC or "not NC." The second or third subclassifiers identified whether the measurement is WM or "not WM," or GBM or "not GBM." Figure 7 shows the ability of each subclassifier to discriminate, for a training group, between $\mathrm{NC}(N=10$, blue), WM $(N=12$, green), and GBM $(N=13$, red) tissue measurements in nine patients. This shows that SLM data can be used to effectively discriminate all three tissue types, provided the classifier is not over trained. The classifier algorithm was tested on eight NC measurements from a different patient with meningioma (Fig. 8).

The near real-time speed of the system makes it much more usable during a clinical trial, with minimal interruption during a surgical procedure. Validation of the TR-FS system in larger trials is still required before technology adoption.

\section{Conclusion}

The field of brain mapping has moved far from just being defined as brain imaging into a field that now includes functional map, brain connectomics, cellular/molecular and metabolomics imaging. This is due to recent advancements in the field of optics and imaging in general, which include molecular and metabolomics imaging/biophotonic/spectroscopy. Biophotonics is being applied more and more to resolve neurological problems, creating the burgeoning new field of neurophotonics. Today the entire field of intraoperative brain mapping is moving toward real time, biomarker-based, meta-data analyticsbased assessment of tissues because the field of neurosurgery in general is moving away from use of major devices and invasive approach to personalized nanoscale diagnostics and therapeutics. Therefore, such advancements in the field of drug discovery/nanoneuroscience, neurophotonics, genomics, and metabolomics are providing enormous opportunities for introduction of intraoperative tools thar are extraordinarily high-resolution capable of visualizing cell(s), real time (up to few nanosecond), and biomarker based. This has revolutionized the field of microscopy and enabled neurosurgeons and physicians to better map the human brain in real time.

Moreover, Raman, fluorescence, and absorption spectroscopy are sensitive to different aspects of molecular and atomic structure and can provide complementary information about tissue characteristics. Multimode systems that combine these techniques may provide even greater sensitivity and specificity for brain tumor demarcation and intraoperative surgical guidance. Every technology has its own limitations but these technologies "together" have great potential to unlock the mysteries of human brain function and disorders. Understanding of basic principles of these technologies is absolutely critical for clinical neuroscientists because knowing the basics will help the scientist to design a personalized therapeutic approach(es) for each patient. The authors of this review strongly believe that integration of neurophotonics with traditional imaging such as ultrasound, CT, MRI, FMRI, device, nanotechnology, cellular therapeutics, supercomputing, artificial intelligence, which is called "nano-bio-electronic" approach will revolutionize the way neurosurgical cases are managed and treated in the future. This team is already moving toward that direction to introduce smart microscopes.

\section{Disclosures}

No conflicts of interest, financial or otherwise, are declared by the authors.

\section{Acknowledgments}

Spectral Molecular Imaging, Inc. (D. L. Farkas, PI) acknowledges support from the US Department of Health and Human Services (under the Qualifying Therapeutic Discovery Program of the Patent Protection and Affordable Care Act of 2010), and by the National Institutes of Health (under NCI SBIR Grant No. 1R44CA183169-01A1). This collaborative project is also made possible by Society for Brain Mapping \& Therapeutics, Brain Mapping Foundation, and National Center for NanoBioElectronics and California Neurosurgical Institute.

\section{References}

1. National Brain Tumor Society, "Quick brain tumor facts," http://braintumor org/brain-tumor-information/brain-tumor-facts/ (8 June 2016).

2. B. Kateb and J. Neale, "Editorial on brain tumor patients, their health insurance and personal bankruptcy," Neuroimage 54, S103-S105 (2011).

3. K. Eder and K. Bernadette, "The dynamics of interactions among immune and glioblastoma cells," Neuromol. Med. Neuromol. Med. 17(4), 335-352 (2015).

4. T. A. Dolecek et al., "CBTRUS statistical report: primary brain and central nervous system tumors diagnosed in the United States in 20052009," Neuro Oncol. 14(Suppl 5), v1-v49 (2012).

5. A. Omuro and L. M. DeAngelis, "Glioblastoma and other malignant gliomas: a clinical review," J. Am. Med. Assoc. 310(17), 1842-1850 (2013)

6. S. Tanaka et al., "Diagnostic and therapeutic avenues for glioblastoma: no longer a dead end?," Nat. Rev. Clin. Oncol. 10(1), 14-26 (2013).

7. E. Albesiano, J. E. Han, and M. Lim, "Mechanisms of local immunoresistance in glioma," Neurosurg. Clin. North Am. 21(1), 17-29 (2010).

8. S. Bao et al., "Stem cell-like glioma cells promote tumor angiogenesis through vascular endothelial growth factor," Cancer Res. 66(16), 78437848 (2006).

9. G. Frosina, "DNA repair and resistance of gliomas to chemotherapy and radiotherapy," Mol. Cancer Res. 7(7), 989-999 (2009).

10. S. Krebs et al., "Genetically modified T cells to target glioblastoma," Front. Oncol. 3, 322 (2013).

11. S. Tanaka et al., "Diagnostic and therapeutic avenues for glioblastoma: no longer a dead end?" Nat. Rev. Clin. Oncol. 10(1), 14-26 (2013).

12. A. Nagy et al., "Mitochondrial energy metabolism and apoptosis regulation in glioblastoma," Brain Res. 1595, 127-142 (2015). 
13. M. Shakarami, A. A. Suratgar, and H. A. Talebi, "Estimation of intraoperative brain shift based on constrained Kalman filter," ISA Trans. 55, 260-266 (2015)

14. O. I. Olubiyi et al., "Intraoperative magnetic resonance imaging in intracranial glioma resection: a single-center, retrospective blinded volumetric study," World Neurosurg. 84(2), 528-536 (2015).

15. G. H. Barnett, "The role of image-guided technology in the surgical planning and resection of gliomas," J. Neurooncol. 42, 247-258 (1999).

16. D. T. Gering et al., "An integrated visualization system for surgical planning and guidance using image fusion and an open MR," J. Magn. Reson. Imaging 13, 967-975 (2001).

17. S. Mori et al., "Brain white matter anatomy of tumor patients evaluated with diffusion tensor imaging," Ann. Neurol. 51, 377-380 (2002).

18. M. H. T. Reinges et al., "Course of brain shift during microsurgical resection of supratentorial cerebral lesions: limits of conventional neuronavigation," Acta Neurochirurgica 146(4), 369-377 (2004).

19. C. Senft et al., "Intraoperative MRI guidance and extent of resection in glioma surgery: a randomised, controlled trial," Lancet Oncol. 12(11), 997-1003 (2011).

20. P. M. Black et al., "Development and implementation of intraoperative magnetic resonance imaging and its neurosurgical applications," Neurosurgery 41(4), 831-845 (1997).

21. C. R. Wirtz et al., "Image-guided neurosurgery with intraoperative MRI: update of frameless stereotaxy and radicality control," Stereotactic Funct. Neurosurg. 68(1-4), 39-43 (1998).

22. F. J. Jolesz, J. Kettenbach, and R. Kikinis, "Image-guided neurosurgery with intraoperative MRI," in Interventional Magnetic Resonance Imaging, pp. 253-260, Springer, Berlin Heidelberg (1998).

23. R. Fahlbusch and A. Samii, "Editorial: intraoperative MRI," Neurosurg. Focus 40(3), E3 (2016).

24. V. M. Gerganov et al., "Two-dimensional high-end ultrasound imaging compared to intraoperative MRI during resection of low-grade gliomas," J. Clin. Neurosci. 18(5), 669-673 (2011).

25. C. R. Wirtz et al., "Image-guided neurosurgery with intraoperative MRI: update of frameless stereotaxy and radicality control," Stereotactic Funct. Neurosurg. 68(1-4), 39-43 (1998).

26. W. A. Hall, H. Liu, and C. L. Truwit, "Functional MRI-guided resection of low grade gliomas," in Int. Congress Series, Vol. 1268, pp. 684-688, Elsevier (2004).

27. W. A. Hall and C. L. Truwit, "Intraoperative MR-guided neurosurgery," J. Magn. Reson. Imaging 27(2), 368-375 (2008).

28. R. Amol et al., "Initial experience of using high field strength intraoperative MRI for neurosurgical procedures," J. Clin. Neurosci. 22(8), 1326-1331 (2015).

29. R. F. Spetzler, J. M. Zabramski, and B. Kaufman, "Clinical role of magnetic resonance imaging in the neurosurgical patient," Neurosurgery 16(4), 511-524 (1985).

30. A. G. Chacko et al., "Intraoperative ultrasound in determining the extent of resection of parenchymal brain tumours-a comparative study with computed tomography and histopathology," Acta Neurochirurgica 145(9), 743-748 (2003).

31. A. K. Petridis et al., "The value of intraoperative sonography in low grade glioma surgery," Clin. Neurol. Neurosurg. 131, 64-68 (2015).

32. M. Woydt et al., "Correlation of intra-operative ultrasound with histopathologic findings after tumour resection in supratentorial gliomas," Acta Neurochirurgica 138(12), 1391-1398 (1996).

33. V. Van Velthoven and L. M. Auer, "Practical application of intraoperative ultrasound imaging," Acta Neurochirurgica 105(1-2), 5-13 (1990).

34. G. Unsgaard et al., "Neuronavigation by intraoperative three-dimensional ultrasound: initial experience during brain tumor resection," Neurosurgery 50(4), 804-812 (2002).

35. J. Sosna et al., "Intraoperative sonography for neurosurgery," $J$. Ultrasound Med. 24(12), 1671-1682 (2005).

36. F. Arlt et al., "Intraoperative 3D contrast-enhanced ultrasound (CEUS): a prospective study of 50 patients with brain tumours," Acta Neurochirurgica 158(4), 685-694 (2016).

37. M. Brant-Zawadzki et al., "Primary intracranial tumor imaging: a comparison of magnetic resonance and CT," Radiology 150(2), 435-440 (1984).

38. Z. Nie et al., "Integrated time-resolved fluorescence and diffuse reflectance spectroscopy instrument for intraoperative detection of brain tumor margin," IEEE J. Sel. Topics Quantum Electron. 22(3), 1-9 (2016).

39. Z. Nie et al., "Hyperspectral fluorescence lifetime imaging for optical biopsy," J. Biomed. Opt. 18(9), 096001 (2013).

40. K. Uluç, G. C. Kujoth, and M. K. Baskaya, "Operating microscopes: past, present, and future," Neurosurg. Focus 27(3), E4 (2009).

41. H. A. Macleod, Thin-Film Optical Filters, CRC press, Chicago (2001)

42. J. G. Fujimoto and D. Farkas, Biomedical Optical Imaging, Oxford University Press, Oxford (2009).

43. P. A. Valdés et al., "Optical technologies for intraoperative neurosurgical guidance," Neurosurg. Focus 40(3), E8 (2016).

44. B. Kateb et al., "Infrared thermal imaging: a review of the literature and case report," Neuroimage 47, T154-T162 (2009).

45. R. D. Ecker et al., "Vision of the future: initial experience with intraoperative real-time high-resolution dynamic infrared imaging: technical note," J. Neurosurg. 97(6), 1460-1471 (2002).

46. A. M. Gorbach et al., "Intraoperative infrared imaging of brain tumors," J. Neurosurg. 101(6), 960-969 (2004).

47. R. Kast et al., "Identification of regions of normal grey matter and white matter from pathologic glioblastoma and necrosis in frozen sections using Raman imaging," J. Neuro-Oncol. 125(2), 287-295 (2015).

48. P. A. Valdés et al., "Optical technologies for intraoperative neurosurgical guidance," Neurosurg. Focus 40(3), E8 (2016).

49. N. Amharref et al., "Discriminating healthy from tumor and necrosis tissue in rat brain tissue samples by Raman spectral imaging," Biochim. Biophys. Acta Biomembranes 1768(10), 2605-2615 (2007).

50. M. Jermyn et al., "Intraoperative brain cancer detection with Raman spectroscopy in humans," Sci. Trans. Med. 7(274), 274ra19-274ra19 (2015).

51. H. Banerjee and M. Verma, "Intraoperative brain cancer detection with Raman spectroscopy in humans," Ann. Trans. Med. 4(4) (2016).

52. M. Wojtkowski et al., "Three-dimensional retinal imaging with high-speed ultrahigh-resolution optical coherence tomography," Ophthalmology 112(10), 1734-1746 (2005).

53. S. A. Boppart et al., "Optical coherence tomography: feasibility for basic research and image-guided surgery of breast cancer," Breast Cancer Res. Treat. 84(2), 85-97 (2004).

54. J. Men et al., "Optical coherence tomography for brain imaging and developmental biology," IEEE J. Sel. Top. Quantum Electron. 22(4), 1-13 (2016).

55. K. Bizheva et al., "Imaging ex vivo healthy and pathological human brain tissue with ultra-high-resolution optical coherence tomography," J. Biomed. Opt. 10(1), 011006 (2005).

56. C. Kut et al., "Detection of human brain cancer infiltration ex vivo and in vivo using quantitative optical coherence tomography," Sci. Trans. Med. 7(292), 292ra100-292ra100 (2015).

57. M. C. Skala et al., "In vivo multiphoton microscopy of NADH and FAD redox states, fluorescence lifetimes, and cellular morphology in precancerous epithelia," Proc. Natl. Acad. Sci. 104(49), 19494-19499 (2007).

58. W. C. Lin et al., "In vivo brain tumor demarcation using optical spectroscopy," Photochem. Photobiol. 73(4), 396-402 (2001).

59. H. J. Lin, P. Herman, and J. R. Lakowicz, "Fluorescence lifetimeresolved pH imaging of living cells," Cytometry Part A 52(2), 77-89 (2003).

60. Q. Liu et al., "Compact point-detection fluorescence spectroscopy system for quantifying intrinsic fluorescence redox ratio in brain cancer diagnostics," J. Biomed. Opt. 16(3), 037004 (2011).

61. Y. G. Chung et al., "Diagnostic potential of laser-induced autofluorescence emission in brain tissue," J. Korean Med. Sci. 12, 135-142 (1997).

62. G. Bottiroli et al., "Brain-tissue autofluorescence: an aid for intraoperative delineation of tumor resection margins," Cancer Detect. Prev. 22, 330-339 (1998).

63. A. C. Croce et al., "Diagnostic potential of autofluorescence for an assisted intraoperative delineation of glioblastoma resection margins," Photochem. Photobiol. 77(3), 309-318 (2003).

64. M. Cardenas-Turanzas et al., "The clinical effectiveness of optical spectroscopy for the in vivo diagnosis of cervical intraepithelial neoplasia: where are we?," Gynecol. Oncol. 107(1), S138-S146 (2007). 
65. M. Y. Berezin and S. Achilefu, "Fluorescence lifetime measurements and biological imaging," Chem. Rev. 110(5), 2641-2684 (2010).

66. P. V. Butte et al., "Fluorescence lifetime spectroscopy for guided therapy of brain tumors," Neuroimage 54, S125-S135 (2011).

67. J. R. Lakowicz, Ed., Principles of Fluorescence Spectroscopy, Springer Science \& Business Media, Berlin (2013).

68. Y. Yuan et al., "High-throughput acousto-optic-tunable-filter-based time-resolved fluorescence spectrometer for optical biopsy," Opt. Lett. 34(7), 1132-1134 (2009).

69. Y. Yuan, T. Papaioannou, and Q. Fang, "Single-shot acquisition of timeresolved fluorescence spectra using a multiple delay optical fiber bundle," Opt. Lett. 33(8), 791-793 (2008).

70. Q. Fang et al., "Time-domain laser-induced fluorescence spectroscopy apparatus for clinical diagnostics," Rev. Sci. Instrum. 75(1), 151-162 (2004).

71. Z. Nie et al., "Hyperspectral fluorescence lifetime imaging for optical biopsy," J. Biomed. Opt. 18(9), 096001 (2013).

72. S. Shrestha et al., "High-speed multispectral fluorescence lifetime imaging implementation for in vivo applications," Opt. Lett. 35(15), 2558-2560 (2010).

73. Y. Sun et al., "Fluorescence lifetime imaging microscopy: in vivo application to diagnosis of oral carcinoma," Opt. Lett. 34(13), 2081-2083 (2009).

74. D. S. Elson, J. A. Jo, and L. Marcu, "Miniaturized side-viewing imaging probe for fluorescence lifetime imaging (FLIM): validation with fluorescence dyes, tissue structural proteins and tissue specimens," New J. Phys. 9(5), 127-127 (2007).

75. Y. Sun et al., "Fluorescence lifetime imaging microscopy for brain tumor image-guided surgery," J. Biomed. Opt. 15(5), 056022 (2010).

76. D. S. Kittle et al., "Real time optical biopsy: time-resolved fluorescence spectroscopy instrumentation and validation," Sci. Rep. 6, 38190 (2016).

77. P. V. Butte et al., "Diagnosis of meningioma by time-resolved fluorescence spectroscopy," J. Biomed. Opt. 10(6), 064026 (2005).

78. P. V. Butte et al., "Fluorescence lifetime spectroscopy for guided therapy of brain tumors," Neuroimage 54, S125-S135 (2011).

79. P. V. Butte et al., "Intraoperative delineation of primary brain tumors using time-resolved fluorescence spectroscopy," J. Biomed. Opt. 15(2), 027008 (2010).
80. F. Vasefi et al., "Intraoperative optical biopsy for brain tumors using spectro-lifetime properties of intrinsic fluorophores," Proc. SPIE 9711, $97111 \mathrm{~T}$ (2016).

Fartash Vasefi, PhD, is a director of Engineering R\&D at SMI Inc. He is interested in commercially adoptable technologies that use multimode optical imaging systems, advanced image processing methods, and integrated micrometer to nanometer scale optical devices in biological sensing and imaging systems. His scientific contribution includes publications in +20 journal articles, +75 conference proceedings, two book chapters, and five US and international patents. He is a senior fellow member of Society for Brain Mapping and Therapeutics.

Nick MacKinnon is VP Product Development at SMI Inc. His interests and focus are on commercialization of cost-effective optical technologies. An experienced entrepreneur, involved with eight medical device startups, and was coinventor of the VELscope, which has screened millions of patients for oral cancer. His background includes over 30 years in development of clinical and research medical devices, working in academic research with the BC Cancer Agency and with several medical device and instrument companies.

Daniel L. Farkas, PhD, a former Fulbright scholar, directed a National Science and Technology Center at Carnegie Mellon University that won the Smithsonian Award for Science. He was a professor of bioengineering, University of Pittsburgh, and vice chairman for research and professor, Department of Surgery, Cedars-Sinai Medical Center. With several prestigious awards, 200+ publications, member of 11 editorial boards, chair of 32 international conferences, his focus is on translational biomedical optical imaging in startups and academia.

Babak Kateb, MD, is a neuroscientist with more than 20 years of research experience. His cross-disciplinary research has been focused on introducing advance and targeted diagnostics and therapeutics to rapidly identify and introduce game-changing technologies to prevent, diagnose, and treat neurological disorders. He is the founding chairman of Society for Brain Mapping and Therapeutics, president of Brain Mapping Foundation, and scientific director and chief strategy officer of California Neurosurgical Institute. 